\title{
The Applied Research on the Virtualization Technology in Cloud Computing
}

\author{
Kun HUANG \\ Computer science and Technology Specialty of Sichuan \\ TOP Vocational Institute \\ Chengdu, China \\ e-mail:83100024@qq.com
}

\begin{abstract}
Cloud computing is emerging as a new business model, it continues to be researched and practiced with. And the virtualization technology is the foundation to achieve cloud computing resource isomerism, pooling and on-demand services. The paper discusses the cloud computing virtualization technology: server virtualization, storage virtualization, network virtualization. Virtualization technology also packages hardware, operating systems, and together with applications into a virtual machine. Then these problems in the cloud computing also are researched: how to deploy quickly a virtual machine, how to optimize the placement of the virtual machine, how to migrate the virtual machine online.
\end{abstract}

Keywords-cloud computing ; applied research;virtualization technology;server virtualization;network virtualization

\section{INTRODUCTION}

Cloud Computing is developed from a distributed computing, parallel computing, grid computing. It is an emerging business model. The goal of cloud computing is to delivered the rich resources to users with "on-demand" approach by the Internet. These resources include those computing resources, storage resources, software development, system testing, system maintenance and the other [1].

Virtualization technology achieves a logical abstraction and unity presentation of network resources, and plays a huge role in the large-scale data center management and solution. It is the most important support of the cloud computing[2].Therefore, the development of cloud computing will inevitably needs more in-depth study on virtualization. This paper will discuss the used virtualization technologies, and how to deploy quickly a virtual machine, how to optimize the placement of virtual machine, how to migrate the virtual machine online.

\section{VIRTUALIZATION TECHNOLOGY FEATURES}

Virtualization technology has the following features: [3]

1) Resource sharing. Package every user's operating environment separately into the virtual machine. This method can realized multi-user sharing of data center resources.

2) Resource customization. Users can take advantage of virtualization technology to configure Server, and specify the number of CPU, memory capacity, disk space of resources according to need.

\author{
Hongjun CHEN \\ Computer science and Technology Specialty of Sichuan \\ TOP Vocational Institute \\ Chengdu, China \\ e-mail:2009missxiaochen@163.com
}

3) Fine-grained resource management. Split a Server into several virtual machines or several physical servers into a virtual machine. It improves server resource utilization, reduce waste, but also helps the server load balancing and energy saving.

Based on the above characteristics, virtualization technology is the basis to achieve resource pooling and ondemand services in the cloud computing.

\section{VIRTUALIZATION IN DIFFERENT LEVELS OF CLOUD COMPUTING}

\section{A. Different virtualization levels}

In cloud computing, virtualized layers are divided into: infrastructure virtualization, platform virtualization, and cloud services. In infrastructure virtualization, server virtualization, storage virtualization, and computer virtualization are important. Application integration, unified network that is network virtualization, unified disaster recovery, unified security guarantees and other technologies are also included. Cloud platforms are deployed on infrastructure virtualization layer.

Cloud platforms include: public cloud, business cloud, and support cloud. Public cloud is that can be accessed through the Internet for public use. Business cloud is that manage networks and services. Support cloud is that includes: BSS- Business Support Systems used to conduct business, such as payment systems; OSS- Operational Support Systems; MSS- Management Support Systems.

Cloud platform provides three different types of user services: IaaS, PssS, and SaaS. IaaS is Infrastructure as a Service that provides services of the use of all facilities to the consumer. These services include handling, storage, networks and other fundamental computing resources. Users can deploy and run arbitrary software, including operating systems and applications. Consumer does not manage or control any cloud computing infrastructure, but it can control the choice of operating system, storage space, deployed applications, there may be limited access to network components (e.g. firewalls, load balancers, etc.) $\mathrm{PaaS}$ is Platform as a Service that provided services of customers using development languages and tools (such as Java, python, .Net, etc.). SaaS is Software as a Service that provides use-on-demand application that run in the cloud platform. See Fig 1. 


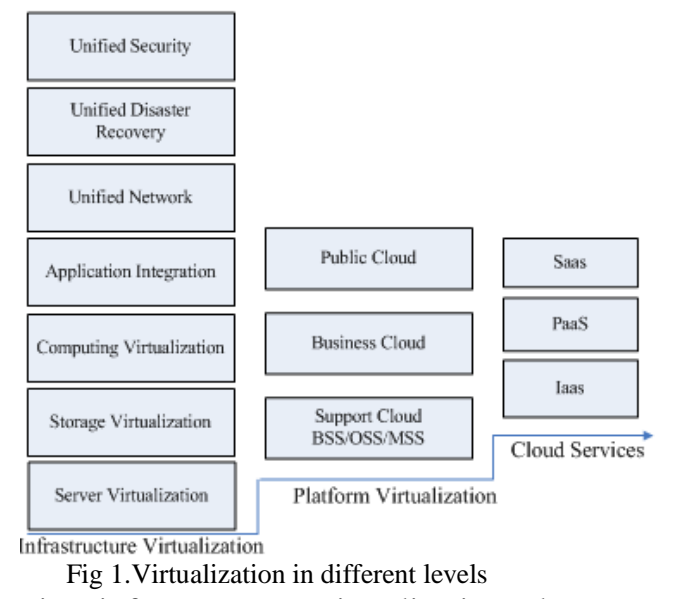

Then, in infrastructure virtualization layer, which technologies are related to server virtualization and storage virtualization and network virtualization?

\section{B. Server virtualization}

Currently, the world's leading server vendors have also introduced a cloud server and virtualization software and other products, such as Citrix's XEN Hypervisor, Microsoft's Hyper-V, Redhat's KVM and Huawei's UVP, etc., which have their own advantages and characteristics in the aspects of virtualization architecture, CPU virtualization , memory virtualization, OS and virtual machine migration, etc. Such as Hyper-V uses a microkernel architecture, code is very small, and does not contain any third-party drivers, so it has high efficiency VMBus structure and perfect support for Linux systems; the KVM is an open source system virtualization module, which uses the Linux scheduler to manage itself, so the core code is smaller than Xen, but KVM requires hardware virtualization support, hardware-based full virtualization.

\section{Storage Virtualization}

Storage virtualization is the idea that separates the logical mapping of resources with its physical storage. The technology improve the hardware utilization in cloud computing storage system, and it take the whole SAN(storage area network) as a storage pool, so obscures the differences between heterogeneous storage, manage and control by the management interface, reduce interoperability, simplify storage allocation and management. Virtual storage technology including snapshot 、 data mirroring replication technology 、 data migration technology and storage consolidation. Data mirroring replication technology is generally based on the hardware but implemented by software are divided into synchronous and asynchronous. According to the used protocol, there are synchronous and asynchronous technologies; data migration technology can migrate data from the old system to a new system. Storage consolidation centralizes capacity allocation, supply, and data movement capabilities to provide greater storage flexibility, reduced cost and system complexity for multi-layer multi-user environment.

\section{Network virtualization}

Virtualized computing and storage resources are ultimately provide services for users in the form of networks. Network architecture can be divided into three parts: the data center network, and across the data center network and ubiquitous cloud access. See Fig 2.

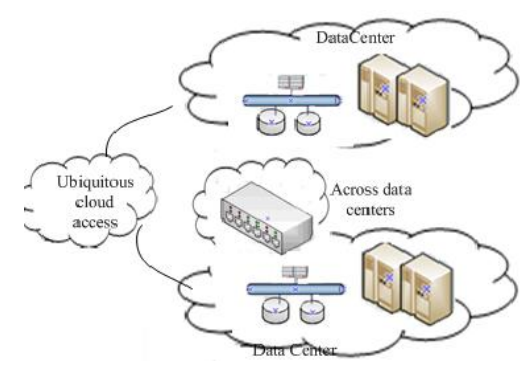

Fig. 2 Network architecture of Data center

Network virtualization includes the core layer and access layer network virtualization and virtual machine swap. The core layer network virtualization refers to the core equipment in core data center, which provide scalable and flexible support; Access layer data center virtualization use hierarchical design, support for new Ethernet technology and a variety of flexible deployment; virtual machine swap form the corresponding function by network switches and physical NICs virtualization. Between data centers compute, or migrate and schedule storage resources by the across-data center. It can build wildly two-layer internet to range the large-scale clustered computing, also build routing networks to satisfy multiple virtual data connection center to provide cloud computing services. In the across -data center, horizontal expansion expands the scale of the data center, while not affecting the network management topology.

Between data centers compute, or migrate and schedule storage resources by the across-data center. It can build wildly two-layer internet to range the large-scale clustered computing, also build routing networks to satisfy multiple virtual data connection center to provide cloud computing services. In the across-data center, horizontal expansion expands the scale of the data center, while not affecting the network management topology.

In addition, cloud computing center provide various services for cloud users by the ubiquitous cloud access network. As cloud users widely distributed, and has large quantities, this ubiquitous cloud access network has strong adaptability. It can provide more secure and reliable cloud data storage security, compatible with multi-ways to access and different terminals. 


\section{DEPLOYMENT OPTIMIZE PLACEMENT AND MIGRATION OF VIRTUAL MACHINES IN THE CLOUD COMPUTING}

Virtualization technologies package hardware, operating systems and applications together into a virtual machine. By adjusting the virtual machine resources, it is easy to realize the scalability of the cloud resources. Then the virtual machine how to deploy, how to optimize placement, and how to migrate it, etc. which should be researched in cloud computing.

\section{A. Rapid Deployment of Virtual Machine Technologies}

Virtual machine template technology is used in most cloud computing platform [5]. Virtual machine templates are preinstalled operating system and application software, and preconfigured virtual devices, so that if can effectively reduce deployment time. However, the virtual machine template technology still can not meet the rapid deployment needs: on the one hand, loading the template into a virtual machine needs to copy the template files. If the files are large, the copy time is relatively more. On the other hand, the preinstalled templates also need to load memory mirroring before providing services. So fork-based thinking of the virtual machine deployment is be researched.

The way can use the parent virtual machine quickly clone a large number of sub-virtual machine. The child can inherit the parent virtual machine's memory status information, and are available immediately after creation. When deploying large-scale virtual machine, the sub-virtual machine can be created parallel, and maintain their separate memory space, rather than depend on the parent virtual machine. To reduce the cost of copying files, virtual machines fork uses a cow- "copy on write" technology: when the sub-virtual machine "writes", the updated file is written to the local disk; when it "reads", firstly it judged whether the file has been updated, if yes, then local disk or parent virtual machine's disk to read the file.

COW key technologies introduce. VM descriptors: a virtual machine descriptor is a concentrated, you can quickly copy a virtual machine to a separate physical host virtual machine images. Virtual machine descriptors construction is started by a kernel thread which lies in virtual machines of static I / O devices. The thread disables all virtual machines CPU, and issues a hypercall to suspend the execution of the virtual machine. See Fig 3.

When the virtual machine call succeeds, function domain( ) mapping process privileges suspends VM memory to fill descriptor. This descriptor contains the following: descriptive metadata for virtual machines and equipment; virtual machines and Xen hypervisor shared by some memory page; main virtual CPU register; memory protection split by X86 used by global descriptor; virtual machine page table, which constitutes a large number of virtual machine description. Each process in the virtual machine usually requires a small additional number of page tables.

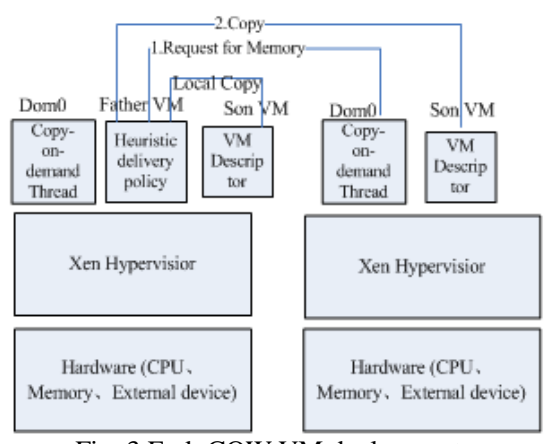

Fig. 3 Fork COW VM deployment

Lagar-Cavilla, who studied Parallel Virtual Machine fork technology based on the distributed environment, which can complete about 32 virtual machines deployment within one second. VM fork is an instant (on-demand) deployment technology, although improving the efficiency of the deployment, but through the deployment of the technology sub virtual machines can not be saved persistently.

\section{B. Virtual Machine Placement Optimization}

Currently, cloud virtual machine placement research work mostly involves only one aspect of optimization: ensure service level objectives, or use the least number of nodes, or reduce costs of the migration of a virtual machine, or reduce energy consumption and so on. However, these optimization targets are mutually conflicting. In order to reduce the number of nodes used, the virtual machine is more compact and placed into a smaller number of nodes, which will turn off idle nodes to save energy consumption and management cost, and need to migrate a virtual machine more often If migration times is reduced, the number of nodes used can be increased. Effective strategy should consider all of these goals and make trade-offs and compromises. So multi-objective optimization and combinatorial optimization are proposed [6].

Firstly, use genetic algorithms to handle virtual machine placement to node in combinatorial optimization problems. And then combine with fuzzy logic to optimize multiple objectives, including the waste of the overall resources, energy consumption and heat dissipation costs are minimized. But the cost of virtual machine migration is not considered. Currently, most virtual machine placement optimization methods are that the multi-objective optimization problem is divided into a number of phases single-objective optimization problem to solve, is rarely performed on multiple targets optimization simultaneously.

\section{Virtual Machine Online MigrationTechnology}

Virtual machine online migration refers that a virtual machine migrates from one physical machine to another physical machine in running station. It has important significance to effectively manage cloud computing platform. It can improve system reliability and conductive load balancing [7]. 
Clark proposes pre-replication (pre-copy) synchronization migration policies in 2005 , but the policy can applied to the cloud limitedly. In order to be used in the cloud, it is necessary to implement system-wide online migration scenarios, which is based on pre-copy synchronous migration strategy [8].

The migration strategy is divided into three phases: push stage, shutdown server to copy stage and pull phase [9]. In the Push phase, the source VM (virtual machine) is still running, and the external memory access is monitored by VMM. Before the memory pre-copy, external memory precopy will execute. After the external memory pre-copy finished, the Xen's memory will start, during the period, external memory access is still monitored by VMM. In the phase of the shutdown to copy, the source VM is hanging up, transfer the remaining memory status, CPU status. And send the record of the VMM monitor to the destination VM. In the pull phase, the destination VM is activated. The destination VM requests data from the source host. For local $\mathrm{I} / \mathrm{O}$ requests the source host as necessary, and meanwhile the source host sending the unsynchronized data in external memory according to pervious state records. See Fig 4.

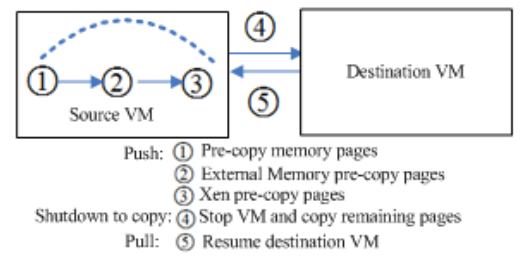

Fig. 4 Pre-copy synchronization migration

\section{VIRTUALIZED ENVIRONMENT TEST}

Experimental platform including 20 physical nodes, each node equipped with two processors which is dual Intel Xeon $3.0 \mathrm{GHz}, 4 \mathrm{~GB}$ RAM, 2 MB L2 Cache, 2 disks which is $7200250 G 13$ SCSI, an Intel Prol000G card.

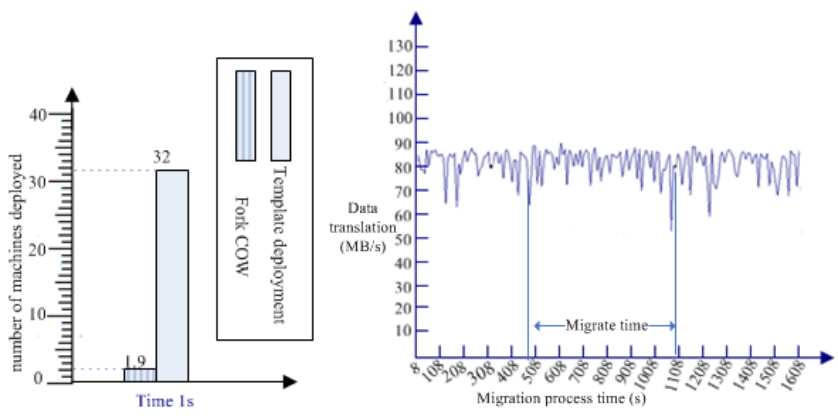

Fig. 5 Compare the efficiency Fig.6 Migration impact on the web

The nodes are connected by a Gigabit Ethernet switch. In addition, 4 nodes as clients send service requests to a virtual machine, generating loads. 2 nodes as a file server, an NFS-based virtual machine disk mirroring. A node as execution algorithm node, which is configured same as the other nodes. In this experiment, the efficiency of the virtual machine deployment and migration of virtual machines are tested.

Experiments show that: Virtual machine templates preinstalled way to deploy a VM, cost time $527 \mathrm{~ms}$ that is deployment about 1.9 VMs within 1 second. See Fig 5. While use COW fork-based proposed by Lagar-Cavilla and others, approximately $32 \mathrm{VMs}$ to be deployed within 1 second. Be seen, a virtual machine can achieve smooth and seamless migration, the migration process has little effect on the data transmission. See fig .6.

So, cloud computing virtualization technology is indispensable technology, and practicable.

\section{SUMMARY}

Virtualization technology is the core technology of cloud computing, it implements that the resources such as the hardware, storage and software, applications, and the other, can allocate them according to need. The rapid deployment of virtual machines, the replacement optimization, and live migration can greatly improve system performance. The adjustment of the virtual machine resources can achieve scalability of software systems, and ensure that the system can quickly recover from a failure and continue to provide services, improve system reliability and stability. Despite the rapid development of cloud computing, it is also faces many problems such as the availability, reliability, security, cost, energy consumption, how to manage their own resources, How to search globally. All the problems are worthy of being researched.

\section{REFERENCES}

[1] Cloud computing, http://baike.baidu.com/view/1316082.htm

[2] VRABLE M, MA J, CHEN J, et al. Scalability, fidelity, and containment in the potemkin virtual honeyfarm [A]. SOSP'05[C] Brighton, United Kingdom: ACM, 2005.148-162.

[3] Menasc D A, Bennani M N Autonomic virtualized environments, 2006.

[4] Junzhou Luo, Cloud Computing: System institutions and key technologies, COMMUNICATIONS, Vol.32 No.7, July 2011

[5] Xu J, Fortes J. Multi-objective virtual machine placement in virtualized data center environments, Proceedings of 2010 IEEE/ACM International Conference on Green Computing and Communications (GreenCom' 2010), 2010: 79-188

[6] Yaqiong Li, Oriented virtualization cloud computing platform memory optimization techniques. Journal of Computers, Vol.34 No.4, Apr.2012

[7] Hines M R, Gopalan K. Post-copy based live virtual machine migration using adaptive pre-paging and dynamic self-ballooning, 2009.

[8] Yuqing Lan. Cloud computing environment online migration technology research, Telecommunications Science, Sept 2010. 